\title{
A characterisation of jointless Dowling geometries
}

\author{
Irasema Sarmiento* \\ Mathematical Institute, Oxford University, 24-29 St. Giles', Oxford OXI 3LB. UK \\ Received 9 July 1997; revised 18 December 1997; accepted 3 August 1998
}

\begin{abstract}
We use statistics of flats of small rank in order to characterise the jointless Dowling geometries defined by groups of order exceeding three and having rank greater than 3. In particular, we show that if the Tutte polynomial of a matroid is identical to the Tutte polynomial of a jointless Dowling geometry, then the matroid is indeed a jointless Dowling geometry. For rank 3 (and groups of order exceeding 3) this holds only if the order of the group is even. (c) 1999 Elsevicr Science B.V. All rights reserved
\end{abstract}

\section{Introduction}

Characterising classes of matroids by their Tutte polynomials is particularly interesting due to the large number of applications of Tutte polynomials [7]. Only a few classes of matroids have been shown to be characterised by their Tutte polynomials. Examples of such classes of matroids are projective and affine geometries and Dowling lattices [4]. We will prove here that jointless Dowling lattices are also characterised by their Tutte polynomials.

Dowling lattices are group-theoretic generalisations of partition lattices $[1,8]$. They have, as well, an interpretation as matroids of gain graphs [14], and are, together with representable matroids, the only non-degenerate varieties [10]. Similarities between projective geometries and Dowling lattices have been studied in $[1,2]$. Dowling lattices form an infinite family of supersolvable tangential blocks [13].

The geometric characterisation of Dowling lattices [4] revolves around a special basis, the elements of which are called joints (these and other Dowling-lattice terms will be defined in Section 2). This was of central importance in [4] for the characterisation of Dowling lattices by statistics of flats of small rank. However, the group structure is reflected only in the lines that do not contain joints. Therefore it is natural to ask whether it is possible to obtain a similar characterisation, by statistics of flats of

\footnotetext{
*E-mail: sarmient@maths.ox.ac.uk. 
small rank, for the geometries obtained from Dowling lattices by deleting the joints. The geometries obtained in this way are the jointless Dowling geometries, and the present paper gives the desired characterisation of these geometries. Jointless Dowling geometries form an infinite family of non-supersolvable tangential blocks [13].

A line in a geometry is a flat of rank 2, a plane is a flat of rank 3. A trivial line is a line having only two points. A trivial plane is a plane having only three points. A trivial rank-r flat is a rank-r flat having only $r$ points.

Let $M$ be a geometry with point set $\mathscr{P}$. We say that $T \subseteq \mathscr{P}$ is line-closed if, for all $\left\{x_{1}, x_{2}\right\} \subseteq T$, the line $x_{1} \vee x_{2}$ spanned by $x_{1}$ and $x_{2}$ is contained in $T$. The geometry $M$ is line-closed if every line closed set in $M$ is a flat of $M$.

A fact of fundamental importance in [1] is that Dowling lattices are line closed. Line closed geometries are characterised by their labelled points and lines. This means that if $N$ is a geometry with the same rank and labelled points and lines as a line closed geometry $M$, then $N$ is equal to $M$. By 'labelled points and lines' we mean labelled points and labelled lines. Line closed geometries were introduced by Halsey in [9], where he proved that supersolvable geometries are line closed. Dowling introduced in [8] the lattices that were later named Dowling lattices and proved that they are supersolvable. Therefore, a Dowling lattice is completely described by the list of its points and lines.

In Section 3 we define the concept of $k$-closure which generalises line-closure. We will prove that $k$-closed geometries are characterised by their flats of rank up to $k$. Furthermore we show that rank-r jointless Dowling geometries over a group $G$ are 4-closed if $r \geqslant 4$. This will allow us to prove in Section 5 that jointless Dowling geometries are characterised by statistics of flats of small rank. The case of rank 3 will be considered in Section 4 .

We will use standard matroid notation as in [12].

\section{Jointless Dowling lattices}

Let $G$ be a finite group (written multiplicatively). The rank-r Dowling lattice over $G$, denoted $Q_{r}(G)$, is the geometry having the following types of points and lines. The points are joints $p_{1}, p_{2}, \ldots, p_{r}$ (which form a basis for $Q_{r}(G)$ ) and internal points $\lambda_{i j}$ (for all $\lambda \in G$ and all $i, j$ positive integers such that $1 \leqslant i<j \leqslant r$ ). The nontrivial lines are coordinate lines $p_{i} \vee p_{j}=\left\{p_{i}, p_{j}\right\} \cup\left\{\lambda_{i j}: \lambda \in G\right\}$ and transversal lines $\left\{\lambda_{i j}, \gamma_{j k},(\lambda \gamma)_{i k}\right\}$ (for all $\lambda, \gamma \in G$ and all $i, j, k$ positive integers with $1 \leqslant i<j<k \leqslant r$ ).

As in [1], Dowling lattices of rank $r \geqslant 4$ can be axiomatically defined through (D1)-(D3) in the following proposition. In the case $r=3$, Dowling lattices based on quasigroups are characterised by these conditions.

Proposition 2.1. A geometry $M$ of rank $r \geqslant 4$ is a Dowling lattice if and only if $M$ has a basis $p_{1}, p_{2}, \ldots, p_{r}$ satisfying the following axioms.

(D1) Each point of $M$ lies on a line $p_{i} \vee p_{j}$. 
(D2) No line $p_{i} \vee p_{j}$ is trivial.

(D3) For points $x \in\left(p_{i} \vee p_{j}\right) \backslash\left\{p_{i}, p_{i}\right\}$ and $y \in\left(p_{i} \vee p_{k}\right) \backslash\left\{p_{i}, p_{k}\right\}$, the line $x \vee y$ is nontrivial.

In the case of a nontrivial group, the basis of $M$ satisfying (D1)-(D3) is unique and consists of the joints. For the trivial group $\langle 1\rangle$, the Dowling lattice $Q_{r}(\langle 1\rangle)$ is the rank-r partition lattice $\Pi_{r+1}$. In this case, we refer to (D1)-(D3) as the axioms of $\Pi_{r+1}$. Dowling lattices can be considered as group-theoretic generalisations of partition lattices.

Let $G$ be a group and let $Q_{r}(G)$ be the Dowling lattice of rank $r \geqslant 2$ based on $G$. Let $p_{1}, \ldots, p_{r}$ be the joints of $Q_{r}(G)$. Then $Q_{r}^{\prime}(G)=Q_{r}(G) \backslash\left\{p_{1} \ldots, p_{r}\right\}$ is called a jointless Dowling geometry.

Note that the points of the jointless Dowling geometry $Q_{r}^{\prime}(G)$ are the internal points $\lambda_{i j}$ of the Dowling geometry $Q_{r}(G)$. The lines of $Q_{r}^{\prime}(G)$ are the transversal lines of $Q_{r}(G)$ and the lines $l_{i j}=\left(p_{i} \vee p_{i}\right) \backslash\left\{p_{i}, p_{j}\right\}$.

The following proposition characterises the non-trivial flats of low rank in jointless Dowling geometries. The characterisation revolves around the lines $l_{i j}$. These special lines are uniquely determined by the first four properties given in the proposition. In Section 3 we will prove that the jointless Dowling lattices are characterised by their labelled flats of rank up to 4 . The main result (Theorem 5.1) of this paper is that a geometry $M$ having the same statistics of flats of rank up to 7 as a jointless Dowling geometry is itself a jointless Dowling geometry. In order to prove it we will label the flats of rank up to 4 of $M$ as those of a jointless Dowling geometry. First we will label a collection of lines of $M$ in such a way that the lines, planes and rank-4 flats of $M$ are precisely as described in Proposition 2.2. Later we will label the points of $M$ as those of a jointless Dowling geometry $Q_{r}^{\prime}(G)$, proving that the labelled flats, of rank up to 4 , of $M$ and $Q_{r}^{\prime}(G)$ coincide. With this we will conclude that $M$ equals $Q_{r}^{\prime}(G)$.

Proposition 2.2. Let $G$ be a group of order at least 4. The jointless Dowling geometry $Q_{i}^{\prime}(G)$ has exactly one set of $\left(\begin{array}{l}r \\ 2\end{array}\right)$ lines $l_{i j}$, with $1 \leqslant i<j \leqslant r$, satisfying the following four properties. (In each statement in this proposition, indices represented by distinct variables are unequal.)

(JD1) The lines $l_{i j}$ partition the points of $Q_{r}^{\prime}(G)$.

(JD2) No line $l_{i j}$ is trivial.

(JD3) For all $x \in l_{i j}$ and $y \in l_{i k}$, the line $x \vee y$ is nontrivial. Furthermore, all nontrivial lines of $Q_{r}^{\prime}(G)$ other than the lines $l_{i j}$ are of this type.

(JD4) For all $i, j, k$, the union of $l_{i j}, l_{i k}$ and $l_{j k}$ is a plane.

The following are the three other types of nontrivial planes of $Q_{r}^{\prime}(G)$.

(JD5) For any point $y$ in $l_{h i}$, the set $l_{j k} \cup y$ is a plane.

(JD6) For points $x$ in $l_{i j}, y$ in $l_{i k}$, and $z$ in $l_{s t}$, the set $(x \vee y) \cup z$ is a plane.

(JD7) For each pair $h, i \in\{j, k, m, n\}$, let $y_{h i}$ be a point in $l_{h i}$, and let $P$ be the sei of these six points. If $P$ satisfies the axioms of $I_{4}$, then $P$ is a plane. 
The following are the nontrivial rank-4 flats of $Q_{r}^{\prime}(G)$.

(JD8) The union of six lines $l_{i j}$ whose indices $i, j$ are the 2-subsets of a 4-element subset of $\{1, \ldots, r\}$ is a rank-4 flat.

(JD9) Let $P$ be a plane as described in (JD4) and $y$ be a point on the line $l_{i j}$. Then $P \cup y$ is a rank-4 flat if neither $i$ nor $j$ are indices of any of the lines in $P$.

(JD10) Let $x$ be a point in $l_{j k}$ and $y$ a point in $l_{m n}$. Then the set $l_{h i} \cup x \cup y$ is a rank-4 flat.

(JD11) Let $x$ and $y$ be points in $l_{i j}$ and $l_{i k}$ respectively. Then the set $l_{m n} \cup(x \vee y)$ is a rank-4 flat.

(JD12) For all $w \in l_{h i}, x \in l_{h j}, y \in l_{k m}$ and $z \in l_{n s}$, the set $(w \vee x) \cup y \cup z$ is a rank-4 flat.

(JD13) For all $w \in l_{h i}, x \in l_{h j}, y \in l_{k m}, z \in l_{k n}$, the set $(w \vee x) \cup(y \vee z)$ is a rank-4 flat.

(JD14) For each pair $h, i$ of elements of $\{j, k, m, n\}$, let $x_{i j}$ be a point of $l_{i j}$ so that the six points $x_{i j}$ satisfy the axioms of $\Pi_{4}$. Then for all $y \in l_{s t}$, the set $\left\{x_{i j}\right\} \cup y$ is a rank-4 flat.

(JD15) For each pair $h, i$ of elements of $\{j, k, m, n, s\}$ let $y_{h i}$ be a point of $l_{h i}$ so that the ten points $y_{h i}$ satisfy the axioms of $\Pi_{5}$ (the partition lattice of rank 4 ). Then these ten points form rank-4 flat.

Proof. Conditions (JD1)-(JD3) are straightforward to prove from the definitions of Dowling and jointless Dowling geometries. If $x$ is a point in $l_{i j}$, then $l_{i k} \vee x$ is a flat as described in (JD4), that is $p_{i} \vee p_{j} \vee p_{k}$. If $y_{j k}, y_{j m}, y_{j n}$ are points in $l_{j k}, l_{j m}$ and $l_{j n}$, respectively, then $\left(y_{j k} \vee y_{j m}\right) \vee y_{j n}$ is a plane $P$. The points $y_{j k}, y_{j m}, y_{j n}$ constitute a basis of $P$ that satisfies the axioms of $\Pi_{4}$. The proof of the remaining conditions follows the same idea, that is, if $F$ is a flat of rank $s$ and $x$ is a point not in $F$, then $F \vee x$ is a flat of rank $s+1$.

Given $x \in l_{i j}$ and $y \in l_{i k}$, the line $x \vee y$ is contained in the plane $l_{i j} \cup l_{i k} \cup l_{j k}$. Therefore $x \vee y$ is a 3-point line with the third point in $l_{j k}$. Fix a point $x$ in $l_{i j}$ and define the function $\phi$ from $l_{i k}$ to $l_{j k}$ where $\phi(y)$ is the third point on the line $x \vee y$ (that is $\phi$ is a projection). Thus all the lines $l_{s t}$ have the same cardinality. Since $Q_{r}^{\prime}(G)$ has $\left(\begin{array}{c}r \\ 2\end{array}\right) g$ points (where $g$ is the order of $G$ ) and there are $\left(\begin{array}{l}r \\ 2\end{array}\right)$ lines in the given collection, all those lines have $g$ points. Assume that $\left\{l_{s t}^{\prime}\right\}$ is a collection of lines satisfying (JD1)(JD4). As above, the lines $l_{s t}^{\prime}$ have $g>3$ points, thus $l_{s t}^{\prime}$ can not be a line of the type described in JD2). Therefore $l_{s t}^{\prime}$ is in $\left\{l_{i j}\right\}$. Since there are $\left(\begin{array}{l}r \\ 2\end{array}\right)$ lines in each collection $\left\{l_{s t}^{\prime}\right\}$ and $\left\{l_{i j}\right\}$, both collections are equal.

\section{3. $k$-closure}

The concept of line-closure is crucial to the proof that the Dowling geometries are characterised by a small collection of counting facts [4]. Unfortunately it is not possible to use line-closure in order to characterise jointless Dowling geometries. 
Proposition 3.1. The only line-closed jointless Dowling geometries are $Q_{2}^{\prime}(G), Q_{3}^{\prime}(G)$ and $Q_{r}^{\prime}(\langle 1\rangle)$, where $G$ is any group and $r \geqslant 2$ is a positive integer.

Proof. Note that $Q_{2}^{\prime}(G)$ has rank 2, therefore it is line-closed. The geometry $Q_{r}^{\prime}(\langle 1\rangle)$ is line-closed because it is isomorphic to $Q_{r-1}(\langle 1\rangle)$.

Recall that $Q_{3}^{\prime}(G)$ has only coordinate and transversal lines and no trivial lines if $g \geqslant 3$. Note that the transversal lines of $Q_{r}^{\prime}(G)$ and $Q_{r}(G)$ coincide. To distinguish joins in $Q_{r}(G)$ from those in $Q_{r}^{\prime}(G)$, we will use $\vee$ for joins in $Q_{r}(G)$ and $\vee^{\prime}$ for those in $Q_{r}^{\prime}(G)$. We will use $\mathrm{Cl}$ to denote the closure in $Q_{r}(G)$ and $\mathrm{Cl}^{\prime}$ to denote the closure in $Q_{r}^{\prime}(G)$

Let $P$ be the set of points of $Q_{3}(G), B=\left\{p_{1}, p_{2}, p_{3}\right\}$ and $P^{\prime}=P \backslash B$ (the set of points of $Q_{3}^{\prime}(G)$ ). Let $T \subseteq P^{\prime}$ be line-closed in $Q_{3}^{\prime}(G)$. If $r(T) \leqslant 2$, then it is clearly a flat of $Q_{3}^{\prime}(G)$. Thus we can assume that $T$ has rank 3. Therefore, there are points $x$ and $y$ in $T$ that lie in two distinct coordinate lines. Since $T$ is line-closed, it contains the 3-point line $x V^{\prime} y$ and it has at least one point in each coordinate line. Since the rank of $T$ is 3 , it has a point that is not on the line $x \vee^{\prime} y$. Thus there is a coordinate line $p_{i} \vee p_{\text {; }}$ that contains two points of $T$. The line $p_{i} \vee^{\prime} p_{j}=\left(p_{i} \vee p_{j}\right) \backslash\left\{p_{i}, p_{i}\right\}$ is contained in $T$ (because $T$ is line-closed). Note that $T$ has a point not in $p_{i} \vee^{\prime} p_{i}$ and the transversal lines of $Q_{3}^{\prime}(G)$ are the same as the transversal lines of $Q_{3}(G)$. Therefore, $T=P^{\prime}$ and $Q_{3}^{\prime}(G)$ is line-closed.

Now let $r \geqslant 4$ and $g \geqslant 2$. We will prove that $Q_{r}^{\prime}(G)$ is not line-closed. Let $A$ be the internal points on the coordinate lines $p_{1} \vee p_{2}$ and $p_{3} \vee p_{4}$. More formally, $A=\left\{\lambda_{12}: \lambda \in G\right\} \cup\left\{\lambda_{34}: \lambda \in G\right\}=\left[\left(p_{1} \vee p_{2}\right) \backslash\left\{p_{1}, p_{2}\right\}\right] \cup\left[\left(p_{3} \vee p_{4}\right) \backslash\left\{p_{3}, p_{4}\right\}\right]$. If $\lambda_{,} \lambda^{\prime}$ are elements of $G$ (not necessarily distinct), then $\lambda_{12} \vee \lambda_{34}^{\prime}=\lambda_{12} \vee^{\prime} \lambda_{34}^{\prime}$ is a trivial line contained in $A$. Furthermore, if $\lambda \neq \lambda^{\prime}$, then $\lambda_{12} \vee^{\prime} i_{12}^{\prime}=\left(p_{1} \vee p_{2}\right) \backslash\left\{p_{1}, p_{2}\right\} \subseteq A$. That $\lambda_{34} V^{\prime} \lambda_{34}^{\prime}$ is contained in $A$ is proved in an analogous way. So $A$ is a line-closed subset of $Q_{r}^{\prime}(G)$. Note that if $\lambda \neq \lambda^{\prime}$, then $\lambda_{12} \vee \lambda_{12}^{\prime}=p_{1} \vee p_{2}$ and $\lambda_{34} \vee \lambda_{34}^{\prime}=p_{3} \vee p_{4}$. Thus the closure of $A$ contains the union of the lines $p_{1} \vee p_{2}$ and $p_{3} \vee p_{4}$, and, in particular, it contains the joints $p_{1}$ and $p_{3}$, hence the line $p_{1} \vee p_{3}$. So $\left(p_{1} \vee p_{3}\right) \backslash\left\{p_{1}, p_{3}\right\} \subseteq \mathrm{Cl}(A)$ $\left\{\left\{p_{1}, \ldots, p_{r}\right\}=\mathrm{Cl}^{\prime}(T)\right.$, but $\left(p_{1} \vee p_{3}\right) \backslash\left\{p_{1}, p_{3}\right\}$ is not contained in $A$. Thus $A \neq \mathrm{Cl}^{\prime}(A)$ and $A$ is not a flat of $Q_{r}^{\prime}(G)$. Therefore, $Q_{r}^{\prime}(G)$ is not line-closed.

Definition 3.2. Let $M$ be a geometry with point set $P$ and let $k$ be a positive integer. We say that $T \subseteq P$ is $k$-closed if for all $\left\{x_{1}, \ldots, x_{k}\right\} \subseteq T, x_{1} \vee \cdots \vee x_{k} \subseteq T$. A geometry $M$ is $k$-closed if every $k$-closed set in $M$ is a flat of $M$.

Note that for every geometry $M$ and for each positive integer $k$, every flat $F$ of $M$ is $k$-closed.

By an argument analogous to that in the proof of Proposition 3.1, the jointless Dowling geometry $Q_{r}^{\prime}(G)$ is not 3-closed when $r \geqslant 4$ and $G$ is a group of order at least 2. If the order of $G$ is one, then $Q_{r}^{\prime}(G)$ is isomorphic to $Q_{r-1}(G)$, thus it is line-closed and, therefore, it is 3-closed. If $r \geqslant 3$, then $Q_{r}^{\prime}(G)$ is trivially 3-closed. 
Proposition 3.3. For all $r \geqslant 4$ and all groups $G$, the jointless Dowling geometry $Q_{r}^{\prime}(G)$ is 4-closed.

Proof. The geometry $Q_{r}^{\prime}(\langle 1\rangle)$ is line closed because it is isomorphic to $Q_{r-1}(\langle 1\rangle)$. Therefore $Q_{r}^{\prime}(\langle 1\rangle)$ is 4-closed.

Let $G$ be a group of order $g \geqslant 2$ and $T^{\prime}$ be a 4-closed set in $Q_{r}^{\prime}(G)$ and $T=T^{\prime} \cup\left\{p_{i}\right.$, $\left.p_{j}:\left(p_{i} \vee p_{j}\right) \backslash\left\{p_{i}, p_{j}\right\} \subseteq T\right\}$. We claim that $T$ is line-closed in $Q_{r}(G)$. If $r\left(T^{\prime}\right)=1$, then both $T$ and $T^{\prime}$ consist of one point. If $r\left(T^{\prime}\right)=2$, then $T$ is a coordinate line or a transversal line or a trivial line depending on whether $T^{\prime}$ contains two points in a coordinate line. Assume that $r\left(T^{\prime}\right) \geqslant 3$ and let $x, y$ be two points in $T$. We need to prove that the line $x \vee y$ is contained in $T$. This can be done by considering the following cases: $x=p_{i}, y=p_{j} ; x=p_{i}, y=\lambda_{i j}$ for some $\lambda \in G ; x=p_{i}, y=\lambda_{j m}$ where $i, j, m$ are distinct; $x=\left(\lambda_{1}\right)_{i j}, y=\left(\lambda_{2}\right)_{i j} ; x=\left(\lambda_{1}\right)_{i j}, y=\left(\lambda_{2}\right)_{i m}$, where $i, j, m$ are distinct, $x=\left(\lambda_{1}\right)_{i j}, y=$ $\left(\lambda_{2}\right)_{k m}$, where $i, j, k, m$ are distinct. In each case checking is easy. Thus $T$ is line-closed, therefore it is a flat of $Q_{r}(G)$. Since $T^{\prime}=T \backslash\left\{p_{1}, \ldots, p_{r}\right\}$ we conclude that $T^{\prime}$ is a flat of $Q_{r}^{\prime}(G)$.

As one can see in the proof of Proposition 3.3, the fact that $Q_{r}(G)$ is line-closed has central importance in proving that $Q_{r}^{\prime}(G)$ is 4-closed.

Proposition 3.4. Let $M$ and $N$ be geometries of rank $r$ such that their rank- $(k+1)$ truncations are isomorphic. If either $M$ or $N$ is $k$-closed, then $N$ and $M$ are isomorphic.

Proof. Without loss of generality we can assume that $N$ is $k$-closed. Let the isomorphism between the truncations $T^{r-k-1}(N)$ and $T^{r-k-1}(M)$ be given by the bijection $\phi$ from $E(N)$ to $E(M)$. Thus, for all $x_{1}, \ldots, x_{k}$ in $E(N)$, we have $y \in x_{1} \vee \cdots \vee x_{k}$ if and only if $\phi(y) \in \phi\left(x_{1}\right) \vee \cdots \vee \phi\left(x_{k}\right)$. Let $F$ be a flat of $M$. Then $F$ is $k$-closed in $M$ and so $\phi^{-1}(F)$ is $k$-closed in $N$. Therefore, $\phi^{-1}(F)$ is a flat of $N$ since $N$ is $k$-closed. Thus, $\phi$ is a bijective strong map between matroids of the same rank. It follows that $M$ and $N$ are isomorphic [11].

\section{The rank-3 case}

By Proposition 3.1, $Q_{3}^{\prime}(G)$ is line-closed. Thus these geometries are characterised by their points and lines, that is, if $N$ is a geometry with the same labelled points and lines as $Q_{3}^{\prime}(G)$, then $N$ is equal to $Q_{3}^{\prime}(G)$.

We say that a flat $F$ of rank $k$ in a geometry is maximal if $F$ is of maximum cardinality among all flats of rank $k . F$ is submaximal if the only flats of rank $k$ of cardinality greater than the cardinality of $F$ are maximal.

Note that if the order of $G$ exceeds three, then the jointless Dowling geometry $Q_{3}^{\prime}(G)$ has exactly three maximal lines and these are disjoint. In the remainder of the paper we will assume that $G$ is a group of order $g>3$. 
The Tutte polynomial of a matroid $M$ on the ground set $S$ is defined by

$$
t(M ; x, y)=\sum_{X \subseteq S}(x-1)^{r(S)-r(X)}(y-1)^{|X|-r \mid Y)} .
$$

From $t(M ; x, y)$ one can determine, among other things, the rank of $M$ and the number of copoints. When $M$ is a rank-3 geometry we can express the number of lines of each cardinality as a linear combination of the coefficients of the Tutte polynomial. For information about the Tutte polynomial, see $[6,7]$.

Theorem 4.1. Let $M$ be a geometry. If $t(M ; x, y)=t\left(Q_{3}^{\prime}(G) ; x, y\right)$ for a group $G$ of even order $g$, then $M$ is a jointless Dowling geometry:

Proof. Since $t(M ; x, y)=t\left(Q_{3}^{\prime}(G) ; x, y\right)$, we have that $M$ has three maximal lines (with $g$ points); let these be $l_{a}, l_{h}, l_{c}$. All other lines of $M$ have exactly three points. We will prove that the maximal lines do not intersect and that $M$ is a jointless Dowling geometry.

Assume that the intersection of the three maximal lines of $M$ is a single point and let $l_{a}=\left\{q, a_{1}, \ldots, a_{q-1}\right\}, l_{b}=\left\{q, b_{1}, \ldots, b_{q j-1}\right\}, l_{c}=\left\{q, c_{1}, \ldots, c_{q j-1}\right\}$. There are two points that are not in any of the maximal lines. Let those points be $q_{1}, q_{2}$. Note that the line $q \vee q_{1}$ cannot contain any point, other than $q$, in the maximal lines. So $q \vee q_{1}=\left\{q, q_{1}, q_{2}\right\}$.

Note that since $q_{1}$ is not on any of the three maximal lines and there are no trivial lines, all lines through $q_{1}$ are 3-point lines. Thus each line through $q_{1}$ other than $\left\{q, q_{1}, q_{2}\right\}$ contains one point from two of the sets $\left\{a_{1}, a_{2}, \ldots, a_{q-1}\right\},\left\{b_{1}, b_{2}, \ldots, b_{1},-1\right\}$, and $\left\{c_{1}, c_{2}, \ldots, c_{s j-1}\right\}$. Furthermore, each point in these three sets is in such a 3-point line through $q_{1}$. Thus the lines through $q_{1}$ pair off the elements in these three sets. However, this is impossible since there are an odd number (specifically. $3 g-3$ ) of elements in these sets. This contradiction shows that there is no point $q$ common to $l_{t,}, l$, and $l_{i}$.

If exactly two of the maximal lines intersect, let $l_{a}=\left\{q, a_{1}, \ldots, a_{q}-1\right\}, \quad l:=$ $\left\{q, b_{1}, \ldots, b_{q-1}\right\}$ and $l_{c}=\left\{c_{1}, \ldots, c_{q q}\right\}$. There is one point that is not in any of the maximal lines, let it be $q_{1}$. For every $i$, the line $q \vee c_{i}$ cannot contain any point from any of the sets $l_{a} \backslash\{q\}, l_{b} \backslash\{q\}$. Since $q \vee c_{i}$ is a 3-point line, it is equal to $\left\{q, q 1-c_{i}\right\}$. However, this is not possible for two different values of $i$.

If exactly two pairs of maximal lines intersect, let the maximal lines of $M$ be $l_{c t}=\left\{q_{1}, a_{1}, \ldots, a_{q-1}\right\}, l_{b}=\left\{q_{1}, b_{1}, \ldots, b_{q-2}, q_{2}\right\}, l_{c}=\left\{q_{2}, c_{1}, \ldots, c_{y-1}\right\}$. Let $q_{3}, q_{+}$be the two points that are not in any of the maximal lines. As above, for every $i$, the line $q_{1} \vee c_{i}$ is $\left\{q_{1}, q_{3}, c_{i}\right\}$ or $\left\{q_{1}, q_{4}, c_{i}\right\}$. This is impossible because $g-1 \geqslant 3$.

Assume that the intersection of every pair of maximal lines is non-empty. Let $l_{a}=\left\{q_{1}, a_{1}, \ldots, a_{q-2}, q_{2}\right\}, \quad l_{h}=\left\{q_{2}, b_{1}, \ldots, b_{y-2}, q_{3}\right\}, l_{c}=\left\{q_{1}, c_{1}, \ldots, c_{q-2}, q_{3}\right\}$. Let $q_{+}$ $q_{5}, q_{6}$ be the three points not in any of the maximal lines. For each $i$, the line $q_{1} \vee b_{i}$ is $\left\{q_{1}, b_{i}, q_{i}\right\}$ for $j$ in $\{4,5,6\}$. Thus $y-2$ is at most 3 . Since $g$ is even, it is equal to 
four. This would imply that one of the lines $q_{1} \vee q_{j}$ (for $4 \leqslant j \leqslant 6$ ) is trivial, which is a contradiction.

Therefore $l_{a}, l_{b}$ and $l_{c}$ are pairwise disjoint. Then $l_{a} \cup l_{b} \cup l_{c}$ has $3 g$ points. Since $M$ has $3 g$ points we have that every point of $M$ is in exactly one of the maximal lines. Let $l_{a}=l_{12}, l_{b}=l_{13}$ and $l_{c}=1_{23}$.

Let $\mathscr{N}_{1}=\left\{l_{12}, l_{13}, E(M)\right\}$; this is a modular cut of $M$. Therefore, it determines a single-element extension $M_{1}$ of $M$. Note that $M_{1}$ is simple and has rank 3. Let $p_{1}$ be the point added to $M$ in order to obtain $M_{1}$. Define a modular cut of $M_{1}$ by $\mathscr{N}_{2}=\left\{\left(l_{12} \cup p_{1}\right), l_{23}, E\left(M_{1}\right)\right\}$. Let $M_{2}$ be the single-element extension of $M_{1}$ determined by it, where $E\left(M_{2}\right)=E\left(M_{1}\right) \cup p_{2}$. Observe that $M_{2}$ is a rank-3 simple matroid. Let $\mathscr{N}_{3}=\left\{l_{13} \cup p_{1}, l_{23} \cup p_{2}, E\left(M_{2}\right)\right\}$, which is a modular cut of $M_{2}$. Let $M_{3}$ be the extension of $M_{2}$ determined by $\mathcal{N}_{3}$, where $E\left(M_{3}\right)=E\left(M_{2}\right) \cup p_{3}$. Note that $M_{3}$ has rank 3 and is simple.

Note that $\left\{p_{1}, p_{2}, p_{3}\right\}$ is a basis of $M_{3}$ and for all $i, j$ such that $1 \leqslant i<j \leqslant 3$, the line $p_{i} \vee p_{j}$ is equal to $l_{i j} \cup\left\{p_{i}, p_{j}\right\}$. Given $i, j, k$ and points $x$ in $\left(p_{i} \vee p_{j}\right) \backslash\left\{p_{i}, p_{j}\right\}$, $y$ in $\left(p_{i} \vee p_{k}\right) \backslash\left\{p_{i}, p_{k}\right\}$, the line $x \vee y$ is non-trivial because $M$ has no trivial lines. By Proposition 2.1, $M_{3}$ is a Dowling geometry defined by a quasigroup $G$ of order $g$. Since $M=M_{3} \backslash\left\{p_{1}, p_{2}, p_{3}\right\}$ we conclude that $M$ is a jointless Dowling geometry.

When $g$ is odd, there are geometries $M$ such that $t(M ; x, y)=t\left(Q_{3}^{\prime}(G) ; x, y\right)$, where $g$ is the order of $G$, yet $M$ is not a jointless Dowling geometry. The following example of such a geometry $M(g)$ is due to Bonin [3]. Recall from [5] (Proposition 7.11) that the Tutte polynomial of a rank-3 geometry is completely determined by its number of points and lines. Thus we need only to construct a geometry on $3 g$ points for which there are three lines having $g$ points, $g^{2}$ lines having three points, and no other lines. The $3 g$ points $M(g)$ lie on four lines through a fixed point $q$, namely $l_{a}=\left\{q, a_{1}, a_{2}, \ldots, a_{g-1}\right\}, l_{b}=\left\{q, b_{1}, b_{2}, \ldots, b_{g-1}\right\}, l_{c}=\left\{q, c_{1}, c_{2}, \ldots, c_{y-1}\right\}$ and $l_{q}=\left\{q, q_{1}, q_{2}\right\}$. Let $\left\{a_{i}, b_{j}, c_{k}\right\}$ be a line if and only if $k+j$ and $i$ are congruent modulo $g$ where $i, j, k \in\{1,2, \ldots, g-1\}$. Let $\left\{q_{1}, a_{i}, b_{i}\right\},\left\{q_{2}, a_{i}, c_{i}\right\}$ and $\left\{q_{2}, b_{i}, c_{g-i}\right\}$ be lines if $i<g / 2$; if $i>g / 2$, then let $\left\{q_{2}, a_{i}, b_{i}\right\},\left\{q_{1}, a_{i}, c_{i}\right\}$ and $\left\{q_{1}, b_{i}, c_{g-i}\right\}$ be lines. An easy congruence argument shows that any two points from distinct sets $l_{a} \backslash\{q\}, l_{b} \backslash\{q\}, l_{c} \backslash\{q\}$ and $l_{q} \backslash\{q\}$ are on precisely one line. Basic counting shows that the number of lines of each cardinality is as needed. Note that $M$ is not a jointless Dowling geometry since the maximal lines of $M$ intersect at a common point.

\section{Geometries of rank at least four}

Our main result is the following theorem which characterises jointless Dowling geometries by statistics of flats of rank up to 7 . It can be regarded as an analogue of Theorem 3.4 in [4]. 
Theorem 5.1. Let $M$ be a rank $r$ geometry $(r>3)$ and $g>3$ an integer. The geometry $M$ is the jointless Dowling lattice $Q_{r}^{\prime}(G)$ defined by a group $G$ of order $g$ if and only if $M$ has the following properties:

1. The geometry $M$ has $\left(\begin{array}{l}r \\ 2\end{array}\right) g$ points.

2. The geometry $M$ has $\left(\begin{array}{l}r \\ 2\end{array}\right)$ lines with $g$ points, $\left(\begin{array}{l}1 \\ 3\end{array}\right) g^{2}$ lines with 3 points, no other nontrivial lines.

3. The geometry $M$ has $\left(\begin{array}{l}r \\ 3\end{array}\right)$ planes with $3 g$ points, and no other planes with $2 g$ or more points.

4. The geometry $M$ has $\left(\begin{array}{l}r \\ 4\end{array}\right)$ rank-4 flats with $6 g$ points, no other rank-4 flats with $5 g$ or more points and a total of $\left(\begin{array}{l}r \\ 4\end{array}\right)+\left(\begin{array}{c}r \\ 3\end{array}\right)\left(\begin{array}{c}r-3 \\ 2\end{array}\right) g+\left[\left(\begin{array}{l}r \\ 2\end{array}\right)\left(\begin{array}{c}r-2 \\ 3\end{array}\right)+\frac{1}{2}\left(\begin{array}{c}r \\ 2\end{array}\right)\left(\begin{array}{c}r-2 \\ 2\end{array}\right)\left(\begin{array}{c}r-4 \\ 2\end{array}\right)\right] g^{2}+$ $\left[\frac{1}{2}\left(\begin{array}{c}r \\ 3\end{array}\right)\left(\begin{array}{c}3 \\ r-3\end{array}\right)+\frac{1}{2}\left(\begin{array}{c}r \\ 3\end{array}\right)\left(\begin{array}{c}r-3 \\ 2\end{array}\right)\left(\begin{array}{c}r-5 \\ 2\end{array}\right)+\left(\begin{array}{c}r \\ 5\end{array}\right)\left(\begin{array}{c}r-4 \\ 2\end{array}\right)+\left(\begin{array}{c}r \\ 5\end{array}\right)+\frac{1}{4 !}\left(\begin{array}{c}r \\ 2\end{array}\right)\left(\begin{array}{c}r-2 \\ 2\end{array}\right)\left(\begin{array}{c}r-4 \\ 2\end{array}\right)\left(\begin{array}{c}r-6 \\ 2\end{array}\right)\right] g^{+} r a n k-4$ flats.

5. The geometry $M$ has $\left(\begin{array}{l}r \\ 5\end{array}\right)$ rank-5 flats with $10 \mathrm{~g}$ points, $\left(\begin{array}{l}r \\ 4\end{array}\right)\left(\begin{array}{c}r-4 \\ 2\end{array}\right)$ g rank-5 flats with $6 g+1$ points and no other rank-5 flats with $6 g$ or more points.

6. The geometry $M$ has $\left(\begin{array}{l}r \\ 6\end{array}\right)$ rank-6 flats with $15 \mathrm{~g}$ points, and no other rank-6 flats with $14 \mathrm{~g}$ or more points.

7. The geometry $M$ has $\left(\begin{array}{l}1 \\ 7\end{array}\right)$ rank-7 flats with $21 \mathrm{~g}$ points, and no other rank-7 flats with $21 \mathrm{~g}-1$ or more points.

Note that Theorem 5.1 implies that if $M_{1}, M_{2}$ are two rank- $r$ geometries for which (1)-(7) hold, then there are groups $G_{1}, G_{2}$ of order $g$ such that $M_{i}$ is isomorphic to $Q_{r}^{\prime}\left(G_{l}\right) \quad(i=1,2)$. However, $G_{1}$ might not be isomorphic to $G_{2}$. This is because the counting information we have assumed reflects only the order of the group, not its structure.

In this section $M$ will denote a rank $r$ geometry satisfying (1)-(7) in Theorem 5.1.

This section is largely devoted to the proof of Theorem 5.1, which is presented through a series of propositions. We will use Proposition 5.2 to prove in Proposition 5.4 that the maximal rank-4 flats containing four maximal planes are the disjoint union of their maximal lines. In Proposition 5.6 we prove that the same holds for maximal rank5 flats containing five maximal rank-4 flats. In Proposition 5.8 it will be proved that every maximal rank-5 flat contains precisely five maximal rank-4 flats. Proposition 5.95.13 are used to prove in Proposition 5.14 that $M$ is the disjoint union of its maximal lines. This is of central importance in proving Proposition 5.17 that is, the flats of $M$ of ranks 2-4 are precisely as described in conditions (JD1)-(JD15) in Proposition 2.2. Finally, we will use a version of Desargues' theorem (Theorem 5.22) to construct a group $G$ of order $g$ and prove that one can label the points of $M$ as in the jointless Dowling geometry $Q_{r}^{\prime}(G)$. Note that this approach is different from the one used at the end of Section 4 , in which we defined modular cuts in order to add the joints.

In [4] the authors proved that if a geometry $M^{\prime}$ has the same statistics of flats of rank up to 7 as a Dowling geometry, then $M^{\prime}$ is itself a Dowling geometry. In order to prove it they exhibit a basis of $M^{\prime}$ that satisfies the conditions (D1) -(D3) in Proposition 2.1. Such a basis is formed by points which are intersection of three maximal planes contained in a maximal rank-4 flat. In fact, in a Dowling 
geometry, the intersection of three maximal planes contained in a maximal rank-4 flat is a joint.

Proposition 5.2. The intersection of three distinct maximal planes contained in a rank-4 flat is empty.

Proof. Let $F$ be a rank-4 flat containing the distinct maximal planes $P_{1}, P_{2}, P_{3}$. Assume that $P_{1} \cap P_{2} \cap P_{3} \neq \emptyset$. As shown in [4], $P_{1} \cap P_{2} \cap P_{3}$ does not contain a line. Thus there is a point $x$ such that $P_{1} \cap P_{2} \cap P_{3}=\{x\}$.

For $i, j$ distinct let $l_{i j}=P_{i} \cap P_{j}$ and $m_{i j}$ be the number of points of $l_{i j} \backslash\{x\}$. Note that $l_{i j}$ is a flat properly contained in $P_{i}$. Thus $l_{i j}$ is a point or a line, hence it has at most $g$ points and so $m_{i j} \leqslant g-1$. Since $P_{i} \backslash\left(l_{i j} \cup l_{i k}\right)=P_{i} \backslash\left[P_{i} \cap\left(P_{j} \cup P_{k}\right)\right]$ and $l_{i j} \cap l_{i k}=P_{i} \cap P_{j} \cap P_{k}=\{x\}$, we have that the union of the three planes $P_{1}, P_{2}$ and $P_{3}$ is

$$
\begin{aligned}
& \{x\} \dot{\cup}\left(l_{12}-x\right) \dot{\cup}\left(l_{13}-x\right) \dot{U}\left(l_{23}-x\right) \dot{\cup}\left[P_{1} \backslash\left(l_{12} \cup l_{13}\right)\right] \dot{\cup}\left[P_{2} \backslash\left(l_{12} \cup l_{23}\right)\right] \\
& \quad \dot{U}\left[P_{3} \backslash\left(l_{13} \cup l_{23}\right)\right] .
\end{aligned}
$$

So the cardinality of $P_{1} \cup P_{2} \cup P_{3}$ is

$$
\begin{aligned}
1 & +m_{12}+m_{13}+m_{23}+\left(3 g-m_{12}-m_{13}-1\right)+\left(3 g-m_{12}-m_{23}-1\right) \\
& +\left(3 g-m_{13}-m_{23}-1\right),
\end{aligned}
$$

that is, $9 g-m_{12}-m_{13}-m_{23}-2$ which is at most $6 g$ ( $F$ has at most $6 g$ points). Thus $3 g-2 \leqslant m_{12}+m_{13}+m_{23} \leqslant 3(g-1)=3 g-3$, and we have a contradiction. Therefore $P_{1} \cap P_{2} \cap P_{3}=\emptyset$.

Proposition 5.3. Each rank-4 flat contains at most four maximal planes. If a rank-4 flat contains four maximal planes, then it is maximal.

Proof. Let $F$ be a rank-4 flat and $P_{1}, P_{2}, P_{3}, P_{4}$ be four maximal planes in $F$. By Proposition 5.2, for all $i, j, k$, we have $P_{i} \cap P_{j} \cap P_{k}=\emptyset$ (here and below distinct subscripts are unequal). Thus

$$
6 g \geqslant|F| \geqslant\left|P_{1} \cup P_{2} \cup P_{3} \cup P_{4}\right|=\sum_{i=1}^{4}\left|P_{i}\right|-\sum_{1 \leqslant i<j \leqslant 4}\left|P_{i} \cap P_{j}\right| \geqslant 12 g-6 g=6 g .
$$

The last inequality is justified because $P_{i} \cap P_{j}$ is a flat of rank at most 2. Thus $F$ and $P_{1} \cup P_{2} \cup P_{3} \cup P_{4}$ have $6 \mathrm{~g}$ points, therefore both sets are equal and $F$ is a maximal rank-4 flat. Moreover $\sum_{1 \leqslant i<j \leqslant 4}\left|P_{i} \cap P j\right|=6 g$. Then for all $i, j$ with $1 \leqslant i<j \leqslant 4$, we have that $P_{i} \cap P_{j}$ has $g$ points and $l_{k m}=P_{i} \cap P_{j}$ is a maximal line. Note that the lines $l_{k m}$ and $l_{k j}$ are disjoint.

Assume that $F$ contains another maximal plane $P_{5}$. For $i=1,2,3,4$ let $l_{i 5}=P_{i} \cap P_{5}$. Note that, $l_{i 5} \cap l_{j 5}=P_{i} \cap P_{j} \cap P_{5}=\emptyset$. As above $l_{i 5}=P_{i} \cap P_{5}$ is a maximal line. Thus 
$3 g=\left|P_{5}\right| \geqslant \sum_{i=1}^{4}\left|P_{i} \cap P_{5}\right|=4 g$, which is a contradiction. Therefore $F$ contains at most four maximal planes. If $F$ contains four maximal planes, then it is a maximal rank-4 flat.

The proofs of Propositions 5.4, 5.5, 5.7 and 5.8 are very similar to the proofs of the analogous propositions in [4]. Only a sketch of the proof of Proposition 5.7 is given: for details see [4].

Proposition 5.4. If a rank-4 flat $F$ contains four maximal planes $P_{1}, P_{2}, P_{3}$ and $P_{4}$. then $F$ is the disjoint union of the six maximal lines $l_{i j}=P_{k} \cap P_{m}$ and these are the only maximal lines of $F$. The plane $P_{i}$ is $l_{j k} \cup l_{m m} \cup l_{k m}(i, j, k, m$, distinct $)$. Each 3-point line of $F$ is in some plane $P_{l}$. Therefore. $F$ contains at most $4 g^{2}$ submaximal lines.

Proof. As in the proof of Proposition 5.3, the lines $l_{i j}$ are maximal and $F$ is a maximal rank-4 flat. By Proposition 5.2 they are disjoint. Since the cardinality of $F$ (namely $6 g$ ) is equal to the sum of the cardinalities of the lines $l_{i j}, F$ is the disjoint union of these lines. For each $i$ let $j, k, m$ be such that $i, j, k, m$ are distinct. Since $l_{i k} \cup l_{j m} \cup l_{k m}$ is a set of cardinality $3 g$ contained in $P_{i}$ (which has cardinality $3 g$ as well). $P_{\text {; }}$ equals $l_{i k} \cup l_{i m} \cup l_{k m}$.

The points of a 3-point line are in different maximal lines, therefore two of these points are in lines $l_{i j}, l_{i k}$ for distinct $i, j, k$. The lines $l_{i j}$ and $l_{i k}$ are contained in $P_{m}$ (with $i, j, k, m$ distinct). Thus two of the points are in $P_{m}$ and so is the given 3-point line. The same argument shows that every maximal line is contained in a maximal plane. It follows that the six lines $l_{i j}$ are the only maximal lines in $F$.

Let us consider the case $r=4$. Since $M$ has $4 q^{2}$ lines with three points, given $x$ in $l_{i j}$ and $y$ in $l_{i k}$ (with $i, j, k$ distinct), by Proposition 5.4, the line $x \vee y$ has three points and the third point is in $l_{j k}$. Note that, since the only planes with cardinality $2 g$ or more are maximal, the rank of $l_{i j} \cup l_{k m}$ is four when $i, j, k, m$ are distinct. If $P$ is a plane of $M$ that contains no maximal line, then $P$ consists of six points one in each maximal line of $M$, the points in $l_{12}, l_{13}$ and $l_{1+}$ are a basis of $P$ which satisfies the axioms for $\Pi_{4}$. One can check that in the case $r=4$ conditions (JDI)-(JD15) in Proposition 2.2 hold for the lines $l_{12}, \ldots l_{34}$. In fact the lines and planes of $M$ are exactly the flats as described in (JD1)-(JD15). We will prove that this holds for $r>4$.

Proposition 5.5. Each rank-5 flat contains at most five maximal rank-4 flats.

Proof. Let $F$ be a rank-5 flat and let $T_{1}, T_{2}, \ldots, T_{1}$ be the maximal rank-4 flats in $F$. Let $i, j$ be positive integers such that $1 \leqslant i<j \leqslant t$. Since $\left|T_{i} \cup T_{j}\right|=12 g-\left|T_{i} \cap T_{j}\right| \leqslant|F| \leqslant 10 \mathrm{~g}$, the cardinality of $T_{i} \cap T_{j}$ is at least $2 g$. Since the maximal lines have $g$ points and the only planes with cardinality at least $2 g$ are maximal, we have that $T_{i} \cap T_{j}$ is a maximal plane. If $T_{1} \cap T_{i}=T_{1} \cap T_{j}$, then $T_{1} \cup T_{i} \cup T_{j}$ has cardinality $12 \mathrm{~g}$. But this is impossible 
because the cardinality of $F$ is at most $10 \mathrm{~g}$. Thus $T_{1}$ is a rank- 4 flat that contains the $t-1$ maximal planes $T_{1} \cap T_{2}, \ldots, T_{1} \cap T_{t}$. By Proposition 5.3 we have that $t-1 \leqslant 4$, therefore $t \leqslant 5$.

Proposition 5.6. Let $F$ be a maximal rank-5 flat containing five maximal rank-4 flats $T_{1}, T_{2}, T_{3}, T_{4}, T_{5}$. Then $F$ is the disjoint union of the ten maximal lines $l_{i j}=T_{k} \cap T_{m} \cap T_{n}$ $(i, j, k, m, n$ distinct $)$ and these are the only maximal lines of $F$. The maximal rank-4 flat $T_{n}$ is the union of the six maximal lines $l_{i j}$ such that $i$ and $j$ are distinct from $n$. All submaximal lines in $F$ lie in planes of the form $l_{i j} \cup l_{i k} \cup l_{j k}$. Hence there are at most $10 g^{2}$ submaximal lines in $F$.

Proof. Each $T_{i}$ contains four maximal planes, the intersection of $T_{i}$ with the other four maximal rank-4 flats. Thus Proposition 5.4 applies to each $T_{i}$. Let $l_{23}, l_{24}, l_{25}, l_{34}, l_{35}, l_{45}$ be the maximal lines of $T_{1}$. We may assume that whenever $\{i, j, m, n\}=\{2,3,4,5\}$, we have $T_{1} \cap T_{m} \cap T_{n}=l_{i j}=l_{j i}$. For $m=2,3,4,5$, we have $T_{1} \cap T_{m}=l_{i j} \cup l_{i n} \cup l_{i n}$ where $i, j, m, n$ are distinct. Considering $T_{2}$, we note that $T_{1} \cap T_{2}=l_{34} \cup l_{35} \cup l_{45}$ and that $T_{2}$ contains three other maximal lines disjoint from them. We label those lines such that

$$
T_{2} \cap T_{3} \cap T_{4}=l_{15}, \quad T_{2} \cap T_{3} \cap T_{5}=l_{14}, \quad T_{2} \cap T_{4} \cap T_{5}=l_{13} .
$$

The four maximal planes contained in $T_{2}$ are correctly labelled (that is for all $m=1,3,4,5$, the intersection of $T_{2}$ and $T_{m}$ is $l_{i j} \cup l_{i n} \cup l_{j n}$ ). Finally, we label the lines in $T_{3}$ such that $l_{12}=T_{3} \cap T_{4} \cap T_{5}$. Thus the five maximal rank four flats contained in $F$ are the union of the required lines.

Given any two of these maximal lines, there is a maximal rank-4 flat that contains both and, by Proposition 5.4, the intersection of the two given lines is empty. Thus $\left|T_{1} \cup T_{2} \cup T_{3}\right|=|F|$ and $F=T_{1} \cup T_{2} \cup T_{3}$. Therefore, $F$ is the disjoint union of the 10 maximal lines $l_{12}, \ldots, l_{45}$.

Let $l$ be a non-trivial line. Then one of the maximal rank-4 flats $T_{j}$ contains $l$ and we can apply Proposition 5.4 to conclude that if $l$ is maximal, then $l \in\left\{l_{12}, \ldots, l_{45}\right\}$ and if $l$ is submaximal, then it is contained in a plane of the form $l_{i j} \cup l_{i k} \cup l_{j k}$ (with $i, j, k$ distinct).

When $r=5, M$ contains five maximal rank-4 flats, therefore Proposition 5.6 applies for $M$. One can easily check that the flats of $M$ of ranks 2-4 are precisely those described in conditions (JD1)-(JD15) in Proposition 2.2.

Proposition 5.7. Each maximal rank-4 flat is in exactly $r-4$ maximal rank-5 flats.

Proof. The first part of the proof consists of proving that every maximal rank- 4 flat is contained in at most $r-4$ maximal rank-5 flats. Let $F$ be a maximal rank-4 flat contained in the maximal rank-5 flats $F_{1}, \ldots, F_{t}$. Then each $A_{i j}=F_{i} \vee F_{j}$ is a maximal rank-6 flat such that the set $L_{i j}=A_{i j}-\left(F_{i} \cup F_{j}\right)$ has cardinality $g$. The sets $L_{i j}$ are pairwise disjoint. There are exactly $6 g+t(10 g-6 g)+\left(\begin{array}{l}t \\ 2\end{array}\right) g$ points in the union of 
$F, F_{1}, \ldots, F_{t}$ and the $\left(\begin{array}{l}t \\ 2\end{array}\right)$ sets $L_{i j}$. Recall that the number of points in $M$ is $\left(\begin{array}{l}t \\ 2\end{array}\right) g$, and thus it follows that $t \leqslant r-4$.

Let

$$
S_{1}, \ldots, S_{(0)}
$$

be all the maximal rank-4 flats of $M$ and $m_{i}$ be the number of maximal rank-5 flats in which $S_{i}$ is contained. There are $\left(\begin{array}{c}r \\ 2\end{array}\right) g-6 g-4 g m_{i}$ submaximal rank-5 flats that contain $S_{i}$. Since at most $\left(\begin{array}{c}r \\ 4\end{array}\right)\left(\begin{array}{c}r-4 \\ 2\end{array}\right) g$ maximal rank-4 flats are contained in submaximal rank-5 flats, we have

$$
\sum_{i=1}^{\left(\begin{array}{c}
r \\
+
\end{array}\right)}\left(\left(\begin{array}{l}
r \\
2
\end{array}\right) g-6 g-4 g m_{i}\right) \leqslant\left(\begin{array}{l}
r \\
4
\end{array}\right)\left(\begin{array}{c}
r-4 \\
2
\end{array}\right) g .
$$

By the first part of the proof $m_{i} \leqslant r-4$, and we have

$$
\left(\begin{array}{l}
r \\
4
\end{array}\right)\left(\left(\begin{array}{l}
r \\
2
\end{array}\right) g-6 g-4 g(r-4)\right) \leqslant\left(\begin{array}{l}
r \\
4
\end{array}\right)\left(\begin{array}{c}
r-4 \\
2
\end{array}\right) g .
$$

We can see, using simple algebra, that the expressions in both sides of this inequality are, in fact, equal. Therefore $m_{i}=r-4$.

Proposition 5.8. Each maximal rank-5 flat contains precisely five maximal rank-4 flats.

Proof. By Proposition 5.5 each maximal rank-5 flat contains at most five maximal rank-4 flats. Therefore, there are at most $5\left(\begin{array}{c}r \\ 5\end{array}\right)$ pairs $\left(F, F^{\prime}\right)$ where $F$ is a maximal rank-5 flat and $F^{\prime}$ is a maximal rank-4 flat contained in $F$. By Proposition 5.7, the number of such pairs is $\left(\begin{array}{l}r \\ 4\end{array}\right)(r-4)$. Since $\left(\begin{array}{l}r \\ 4\end{array}\right)(r-4)=5\left(\begin{array}{l}r \\ 5\end{array}\right)$, we have that each maximal rank-5 flat contains precisely five maximal rank-4 flats.

Therefore Proposition 5.6 applies to all maximal rank-5 flats.

Let $F$ be a maximal rank-4 flat and let $F_{5}, \ldots, F_{r}$ be the $r-4$ maximal rank-5 flats that contain $F$. Since $F_{5}$ contains five maximal rank-4 flats, by Proposition 5.6 the ten maximal lines of $F_{5}$ can be labelled as $l_{12}, l_{13}, \ldots, l_{45}$ so that $F=\bigcup_{i, j \in\{1.2 .3,\}} l_{i j}$ and three maximal lines form a maximal plane if and only if their subscripts come from a 3 -subset of $\{1,2,3,4,5\}$. By Proposition 5.6, this labelling can be extended to a labelling of the maximal lines of each $F_{i}$, for $i \geqslant 5$, so that three maximal lines in $F_{i}$ form a maximal plane if and only if their subscripts come from a 3 -subset of $\{1,2,3,4, i\}$. It follows from this labelling that six maximal lines in $F_{i}$ form a maximal rank-4 flat if and only if their subscripts come from a 4 -subset of $\{1,2,3,4, i\}$. For $5 \leqslant i<j \leqslant r$ let $F_{i j}=l_{12} \vee l_{13} \vee l_{1 i} \vee l_{1 j}$.

Proposition 5.9. $F_{i j}$ is a maximal rank-5 flat. 
Proof. Let $A=l_{12} \vee l_{13} \vee l_{1 i}$ and $B=l_{12} \vee l_{13} \vee l_{1 j}$. Note that $A$ consists of the six maximal lines of $F_{i}$ having subscripts in the 4 set $\{1,2,3, i\}$, therefore $A$ is a maximal rank-4 flat. Similarly $B$ is a maximal rank-4 flat contained in $F_{j}$. Thus $A$ and $B$ are maximal rank-4 flats meeting in the maximal plane $l_{12} \vee l_{13}$ and such that $F_{i j}=A \vee B$. By semimodularity, the rank of $F_{i j}$ is five. Since $\left|F_{i j}\right| \geqslant|A \cup B|=|A|+|B|-|A \cap B|=9 g$ and the only rank-5 flats with cardinality $9 g$ or more are maximal, we have that $F_{i j}$ is a maximal rank-5 flat.

Let $l_{i j}$ be the missing line in $F_{i j}$. That is, $F_{i j}$ is the disjoint union of the ten maximal lines $l_{k m}$ with $k, m$ in $\{1,2,3, i, j\}$. We want to argue that the $\left(\begin{array}{l}r \\ 2\end{array}\right)$ maximal lines $l_{i j}$ partition the points of $M$. Since any two of these maximal lines occur in one of the pairs $F_{i j}$ and $F_{i}, F_{i j}$ and $F_{k}, F_{i j}$ and $F_{i k}$, and $F_{i j}$ and $F_{k m}$, with one line in the first flat and the other in the second, it suffices to show that the intersections of these flats are as small as possible. (Here and below we assume that distinct subscripts are unequal.) Specifically we need to show

$$
F_{i j} \cap F_{i}=l_{12} \vee l_{13} \vee l_{1 i}=F_{i j} \cap F_{i k},
$$

and

$$
F_{i j} \cap F_{k}=l_{12} \vee l_{13}=F_{i j} \cap F_{k m} .
$$

Proposition 5.10. The intersection of $F_{i j}$ and $F_{i}$ is the maximal rank-4 flat $l_{12} \vee l_{13} \vee$ $l_{1}$, that is, the disjoint union of the six maximal lines $l_{k m}$ with $k, m$ in $\{1,2,3, i\}$.

Proof. This is immediate since $F_{i j}$ and $F_{i}$ are unequal rank-5 flats that both contain the rank-4 flat $l_{12} \vee l_{13} \vee l_{1 i}$.

Proposition 5.11. The intersection of $F_{i j}$ and $F_{k}$ is the maximal plane $l_{12} \vee l_{13}$.

Proof. The intersection of $F_{i j}$ and $F_{k}$ has rank 3 or 4 , if it has rank 3 , then it is equal to $l_{12} \vee l_{13}$. In order to prove that the rank of $F_{i j} \cap F_{k}$ is not 4 , it is enough to prove that $r\left(F_{i j} \vee F_{k}\right)$ is not 6. If $F_{i j} \vee F_{k}$ had rank 6, then $|5 g| \geqslant\left|F_{i j} \vee F_{k}\right| \geqslant\left|F_{i j} \cup F_{k}\right|=20 g-$ $\left|F_{i j} \cap F_{k}\right|$. Thus $\left|F_{i j} \cap F_{k}\right| \geqslant 5 g$, which would force $F_{i j} \cap F_{k}$ to be a maximal rank-4 flat in $F_{k}$. This is impossible because every maximal rank-4 flat in $F_{k}$ is the union of the lines $l_{s t}$ with subscripts in a 4 -subset of $\{1,2,3,4, k\}$.

Proposition 5.12. The intersection of $F_{i j}$ and $F_{i k}$ is the maximal rank-4 flat $l_{12} \vee l_{13} \vee l_{1 i}$

Proof. Just note that $F_{i j}$ and $F_{i k}$ are two distinct rank-5 flats containing the rank-4 flat $l_{12} \vee l_{13} \vee l_{1 i}$.

The proof of Proposition 5.13 is similar to the proof of Proposition 5.11. 
Proposition 5.13. The intersection of $F_{i j}$ and $F_{k m}$ equals the maximal plane $l_{12} \vee l_{13}$.

Proposition 5.14. The geometry $M$ is the disjoint union of the maximal lines $l_{i j}$. with $1 \leqslant i<j \leqslant r$.

Proof. Using the previous four propositions it follows that the lines $l_{i j}$ are pairwise disjoint. Therefore the cardinality of $\dot{U}_{1 \leqslant 1<j \leqslant r} l_{i j}$ equals $\sum_{1 \leqslant i<j \leqslant r}\left|l_{i j}\right|=\left(\begin{array}{l}r \\ 2\end{array}\right) g$ which is the number of points of $M$.

The following proposition can be easily checked.

Proposition 5.15. Let $s$ be such that $3 \leqslant s \leqslant 7$, and let $\mathscr{L}$ be a set of maximal lines. The union of the lines in $\mathscr{L}$ is a maximal rank-s flat if and only if their subscripts form an s-subset of $\{1, \ldots, r\}$.

Proposition 5.16. Every submaximal line is contained in a maximal plane $l_{i j} \vee l_{i h}$.

Proof. Let $y_{1}$ and $y_{2}$ be two points in a submaximal (3-point) line. By Proposition $5.14, M$ is the disjoint union of the maximal lines $l_{i j}(1 \leqslant i<j \leqslant r)$. Thus there are $i, j, k, m$ (not necessarily distinct) such that $y_{1} \in l_{i j}$ and $y_{2} \in l_{k m}$. Therefore, the given line is contained in a maximal rank-4 flat, and, by Proposition 5.6 (rank 5 case) it is contained in a maximal plane.

Proposition 5.17. The flats of $M$ of ranks 2-4 are precisely as specified in conditions (JD1)-(JD15) of Proposition 2.2 .

Proof. By Proposition 5.15 the maximal flats of ranks $2-4$ are exactly those described in (JD1), (JD4) and (JD8) in Proposition 2.2. The condition (JD3) follows easily from Proposition 5.16 using that there are $\left(\begin{array}{l}3 \\ 3\end{array}\right) g^{2}$ lines with three points. All sets of points described in (JD5)-(JD15) are contained in the union of a set of maximal lines as in Proposition 5.15. Therefore, those sets of points are contained in a maximal flat of rank at most 7 , it is easy to check that they are flats.

We wish to prove now that there are no other flats of rank 2,3 or 4 . It is easy by considering that a flat of rank $s$ is generated by a flat $F$ of rank $s-1$ and a point not in $F$. In particular, we have to prove that given distinct indices $i, j, k, m, n, t, l, w$ and points $x_{i j}, x_{k m}, x_{m}, x_{i w}$ in $l_{i j}, l_{k m}, l_{n i}, l_{i w}$, the set $\left\{x_{i j}, x_{k m}, x_{m i}, x_{i m}\right\}$ is a rank-4 flat. Only there we use the total number of rank-4 flats.

We will give a geometric construction based on $M$ that produces a group $G$ of order $g$ so that $M$ and $Q_{,}^{\prime}(G)$ have, up to labelling, the same points and lines. In other words, the truncations to rank 3 of $M$ and $Q_{r}^{\prime}(G)$ are isomorphic. From this, Propositions 2.2. and 5.17, it follows that $M$ and $Q_{r}^{\prime}(G)$ have, up to relabelling, the same flats of ranks 2-4. From this, Theorem 4.1 follows immediately since $Q_{r}^{\prime}(G)$ is 4-closed. 
Proposition 5.18. Let $N$ be a geometry of rank $r \geqslant 4$ such that

(JD1) there are $\left(\begin{array}{c}r \\ 2\end{array}\right)$ disjoint lines $l_{i j}(1 \leqslant i<j \leqslant r)$ such that every point lies in exactly one of these lines,

(JD2) no line $l_{i j}$ is trivial,

(JD3) for all $x \in l_{i j}$ and all $y \in l_{i k}$ the line $x \vee y$ is a 3-point line with the third point in $l_{j k}$, and

(JD4) for all $1 \leqslant i<j<k \leqslant r$, the union of the lines $l_{i j}, l_{i k}$ and $l_{j k}$, is a plane.

Then there is a group $G$ such that $N$ has the same (labelled) points and lines as $Q_{r}^{\prime}(G)$.

From now on $N$ will be a geometry as in Proposition 5.18, and we will call the lines in (JD1) coordinate lines and the planes in (JD4) coordinate planes. As in [1] one easily shows that all the coordinate lines have the same number of points.

Definition 5.19. Let two triplets of points $a, b, c$ and $a^{\prime}, b^{\prime}, c^{\prime}$ be given. We say that the triangles $a, b, c$ and $a^{\prime}, b^{\prime}, c^{\prime}$ are in perspective from $i$ if there exists $j, k, m$ such that $a, a^{\prime} \in l_{i j}, b, b^{\prime} \in l_{i k}$ and $c, c^{\prime} \in l_{i m}$.

Definition 5.20. Given three points $a, b, c$, we say that the triangle $a, b, c$ is in perspective from $i$ with the coordinate plane $l_{j k} \vee l_{j m}$ if $a \in l_{i j}, b \in l_{i k}, c \in l_{i m}$.

The concept of perspective from $i$ is based on the concept of perspective from the joint $p_{i}$ in a Dowling lattice. The concept of perspective from a line is analogous to that in Dowling lattices (see [1]).

Definition 5.21. Given three points $a, b, c$ we say that the triangle $a, b, c$ and the coordinate plane $l_{j k} \vee l_{j m}$ are in perspective from a line if $(a \vee b) \wedge l_{j k},(a \vee c) \wedge l_{j m}$, $(b \vee c) \wedge l_{k m}$ are collinear points.

The following can be regarded as a Desargues' theorem for jointless Dowling lattices (Fig. 1).

Theorem 5.22. If a triangle $a, b, c$ is in perspective from $i$ with the coordinate plane $l_{j k} \vee l_{j m}$, then it is in perspective from a line with the given coordinate plane.

Proof. The points $a, b, c$ span a plane $P$. The plane $P$ meets $l_{j k} \vee l_{j m}$ in three points (namely $\left.q=(a \vee b) \cap l_{j k}, p=(a \vee c) \cap l_{j m}, s=(b \vee c) \cap l_{k m}\right)$. Therefore these points lie on a line (the intersection of the two planes).

Corollary 5.23. Let $a, b, c$ and $a^{\prime}, b^{\prime}, c^{\prime}$ be two triangles in perspective from $i$ and such that $a \vee b, a^{\prime} \vee b^{\prime}$ and $b \vee c, b^{\prime} \vee c^{\prime}$ are pairs of intersecting lines. Thus $a, b, c$ and $a^{\prime}, b^{\prime}, c^{\prime}$ are in perspective from a line and, in particular, $a \vee c$ and $a^{\prime} \vee c^{\prime}$ meet in $a$ point. 


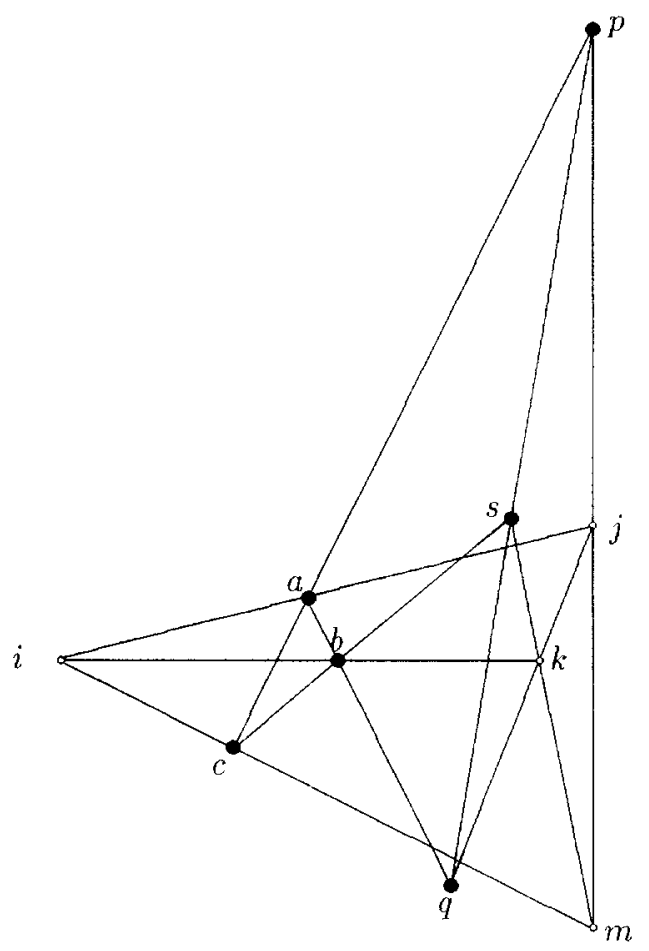

Fig. 1. Desargues' theorem for jointless Dowling lattices.

As in [1] we define a binary operation in each coordinate line of $N$, and prove that this defines a group $G$ and that it is possible to label the points of $N$ as in $Q_{r}^{\prime}(G)$. The binary operation is defined as follows (see Fig. 2).

Fix a point $l$ in $l_{i j}$ and a point $d$ in $l_{i k}$. Let $p$ be the third point in the line $1 \vee d$ (note that $p$ is in $l_{j k}$ ). Let $a, c$ be arbitrary points in $l_{i j}$. These are the points that we will multiply. Let $d^{\prime}$ be the third point in $a \vee p$ and $q$ the third point on $c \vee d$. Then $a c$ is defined to be the third point on the line $q \vee d^{\prime}$.

By Corollary 5.23 the binary operation defined on the lines $l_{i j}$ depends only on the points of $l_{i j}$ and not on the choice of auxiliary points on other lines. As in [1] this operation defines a group, with identity 1 , on the points of each coordinate line. Groups on different coordinate lines are isomorphic by projection. Let $G$ be the group defined on the points of every coordinate line. In order to label the points of $N$ as in $Q_{r}^{\prime}(G)$ fix a point 1 in $l_{1 j}$ (for $j \geqslant 2$ ). For each $j>2$, let $l_{2 j}$ be the third point on the line $1_{12} \vee 1_{1 j}$. Label the points of $l_{12}$ labelling the group $G$. For each $\lambda$ in $G$ and each $j>2$, label as $\lambda_{1 j}$ the third point on the line $\lambda_{12} \vee 1_{2 j}$. For $2 \leqslant i<j \leqslant n$ label as $\lambda_{i j}$ the third point on the line $1_{1 i} \vee i_{1 j}$. Using Corollary 5.23 one shows that the 3-point lines of $N$ are labelled as those of $Q_{r}^{\prime}(G)$. For details see [1].

This proves Proposition 5.18 and therefore Theorem 5.1.

In [6] a more general version of the following proposition is proved. 


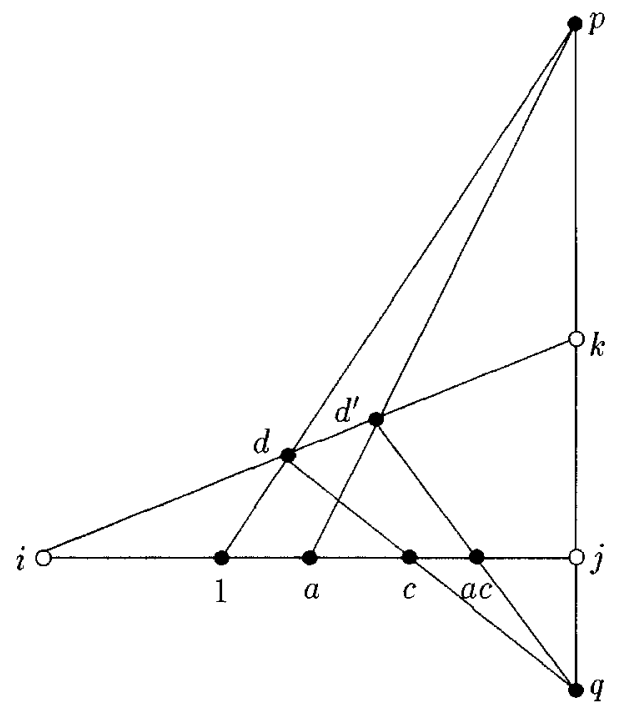

Fig. 2. Binary operation in the line $l_{i j}$.

Proposition 5.24. For a rank-r matroid $R$ and any integer $i$ with $0 \leqslant i \leqslant r$, let $c_{i}$ be the largest cardinality among rank-i flats of $R$. Then for each $i$ with $1 \leqslant i \leqslant r$ and each $j$ with $c_{i-1}<j \leqslant c_{i}$, we can express the number of flats of $R$ having rank $i$ and cardinality $j$ as a linear combination of the coefficients of the Tutte polynomial of $R$.

By Proposition 5.24 and Theorem 5.1 the following holds.

Corollary 5.25. Let $M$ be a matroid such that $T\left(Q_{r}^{\prime}(G) ; x, y\right)=T(M ; x, y)$ for a group $G$ of order $g$. Thus there exists a group $G_{1}$, uniquely determined by the matroid structure of $M$, such that $G_{1}$ has order $g$ and $M$ is isomorphic to the jointless Dowling lattice $Q_{r}^{\prime}\left(G_{1}\right)$.

This proves that jointless Dowling geometries are characterised both by their Tutte polynomial and by the counting information given in Theorem 5.1.

\section{Acknowledgements}

I thank Professor Joseph E. Bonin at George Washington University for introducing me to the problem and for several helpful discussions. I thank my advisor D.J.A. Welsh for his patience, support and guidance. I am grateful to Eric Bartels for his help writing this paper. The work was supported by the Consejo Nacional de Ciencia y Tecnologia of Mexico through Ph.D. scholarship 22059. 


\section{References}

[1] M.K. Bennett, K.P. Bogart, J.E. Bonin. The geometry of Dowling lattices, Adv. Math. 103 (1994) $131-161$.

[2] J.E. Bonin. Automorphisms of Dowling lattices and related geometries. Combin. Probab. Comput. 4 (1995) $1-9$.

[3] J.E. Bonin, Private communication.

[4] J.E. Bonin, W.P. Miller, Characterizing geometries by numerical invariants, Preprint.

[5] T.H. Brylawski, A decomposition for combinatorial geometries, Trans. AMS 171 (1972) 235-282.

[6] T.H. Brylawski, The Tutte polynomial. Part I: General theory, in: A. Barlotti (Ed.). Matroid Theory and Its Applications, C.I.M.E., Liguori, Naples, 1980, pp. 125-175.

[7] T.H. Brylawski, J. Oxley, The Tutte polynomial and its Applications, in: N. White (Ed.), Matroid Applications, Cambridge University Press, Cambridge, 1992, pp. 123-225.

[8] T.A. Dowling, A class of geometric lattices based on finite groups, J. Combin. Theory B 14 (1973) 61-86.

[9] M.D. Halsey, Line-closed combinatorial geometries, Diserete Math. 65 (1987) 245-248.

[10] J. Kahn, J. Kung, Varieties of combinatorial geometries, Trans. Amer. Math. Soc. 271 (1982) $485-499$.

[11] J.P.S. Kung, Strong maps, in: N.L. White (Ed.), Theory of Matroids. Cambridge University Press, Cambridge, 1986, pp. 224-253.

[12] J.G. Oxley, Matroid Theory, Oxford University Press, Oxford, 1992.

[13] G. Whittle. Dowling group geometries and the critical problem. J. Combin. Theory Ser. B 47 (1989) $80-92$.

[14] T. Zaslavsky. Biased graphs. I. Bias, balance, and gains. J. Combin. Theory Ser. B 47 (1989) 3252. 